2. C. J. Everett, Vector spaces over rings, Bull. Amer. Math. Soc. vol. 48 (1942) pp. 312-316.

3. B. L. van der Waerden, Moderne Algebra, vol. 2, 1st ed., Berlin, 1931.

UNIVERSITY OF WISCONSIN

\title{
ON A CONSTRUCTION FOR DIVISION ALGEBRAS OF ORDER 16
}

R. D. SCHAFER

It is not known whether there exist division algebras of order 16 (or greater) over the real number field $\Re$. In discussing the implications of this question in algebra and topology, A. A. Albert told the author that the well known Cayley-Dickson process ${ }^{1}$ does not yield a division algebra of order 16 over $\Re$ and suggested a modification of that process which might. It is the purpose of this note to show that, while Albert's construction can in no instance yield such an algebra over $\Re$, it does yield division algebras of order 16 over other fields, in particular the rational number field $R$.

Initially consider an arbitrary field $F$. Let $C$ be a Cayley-Dickson division algebra of order 8 over $F$. Define ${ }^{2}$ an algebra of order 16 over $F$ with elements $c=a+v b, z=x+v y(a, b, x, y$ in $C)$ and with multiplication given by

$$
c z=(a+v b)(x+v y)=(a x+g \cdot y b S)+v(a S \cdot y+x b)
$$

where $S$ is the involution $x \rightleftarrows x S=t(x)-x$ of $C$ and $g$ is some fixed element of $C$. The Cayley-Dickson process is of course the instance $g=\boldsymbol{\gamma}$ in $F$.

For $A$ to be a division algebra over $F$ the right multiplication ${ }^{1} R_{z}$ must be nonsingular for all $z \neq 0$ in $A$. Now

$$
R_{z}=\left(\begin{array}{cc}
R_{x} & S R_{y} \\
S L_{y} L_{0} & L_{x}
\end{array}\right)
$$

Received by the editors January 19, 1945, and, in revised form, March 19, 1945.

${ }^{1}$ See [1] and [2] for background and notations. Numbers in brackets refer to the references cited at the end of the paper.

2 We should remark that this modification of the Cayley-Dickson process does yield non-alternative division algebras of orders 4 and 8 over $\Re$ when applied to the algebras of complex numbers and real quaternions instead of to $C$. See R. H. Bruck, Some results in the theory of linear non-associative algebras, Trans. Amer. Math. Soc. vol. 56 (1944) pp. 141-199, Theorem 16C, Corollary 1, for a generalization. 
and, if $g \neq 0, R_{z}$ is nonsingular in case either $x=0, y \neq 0$ or $x \neq 0, y=0$. Therefore let $x \neq 0, y \neq 0$. Then

$$
\begin{aligned}
& \left|R_{z}\right|=\left|\begin{array}{cc}
R_{x} & S R_{y} \\
S L_{y} L_{g} & L_{x}
\end{array}\right|=\left|\begin{array}{cc}
R_{x} & 0 \\
S L_{y} L_{0} & L_{x}-S L_{y} L_{g} R_{x}^{-1} S R_{y}
\end{array}\right| \\
& =\left|R_{x}\right| \cdot\left|L_{x}-\frac{1}{n(x)} R_{y S} R_{o S} L_{x} R_{y}\right| \\
& =\left|R_{x}\right| \cdot\left|L_{x}-\frac{1}{n(x)} R_{y S} R_{o S} R_{x}^{-1} R_{x y} L_{x}\right|
\end{aligned}
$$

by a lemma of Moufang. ${ }^{3}$ Hence $\left|R_{z}\right|=\left|L_{x}\right| \cdot\left|n(x) R_{y} R_{g}\right|^{-1}$ . $\left|n(x) R_{x} R_{g} R_{y}-n(g) n(y) R_{x y}\right|$. That is, $A$ is a division algebra over $F$ if and only if the transformation

$$
n(x) R_{x} R_{g} R_{y}-n(g) n(y) R_{x y}
$$

is nonsingular for all $x, y \neq 0$ in $C$.

Now let $F=\Re$, the field of real numbers. The non-scalar ${ }^{4}$ element $g$ generates $B \subset Q=B+u B, Q$ a real quaternion algebra, $u^{2}=-n(u)$, $g u=u \cdot g S$. Multiplication in the Cayley algebra $C=Q+w Q$ is defined by the right multiplication

$$
R_{q+w r}=R_{(q, r)}=\left(\begin{array}{rr}
R_{q} & S R_{r} \\
-S L_{r} & L_{q}
\end{array}\right)
$$

for $q, r$ in $Q$ and $S$ the involution $q \rightleftarrows q S=t(q)-q$ of $Q$. Specialize two elements $x, y$ of $C$ in the following manner. Let $y=u \in Q$; then $y^{2}=-n(y), g y=y \cdot g S$. Let $x \in w Q$ and $n(x)=\zeta n(y)$ where $\zeta>0$, $\zeta^{2}=n(g)$. Then $x y=(0, x) R_{(y, 0)}=(0, y x)$ and

$$
\begin{aligned}
& \left|n(x) R_{x} R_{g} R_{y}-n(g) n(y) R_{x y}\right| \\
& =\left|n(x)\left(\begin{array}{cc}
0 & S R_{x} \\
-S L_{x} & 0
\end{array}\right)\left(\begin{array}{cc}
R_{g} & 0 \\
0 & L_{g}
\end{array}\right)\left(\begin{array}{cc}
R_{y} & 0 \\
0 & L_{y}
\end{array}\right)-n(g) n(y)\left(\begin{array}{cc}
0 & S R_{y x} \\
-S L_{y x} & 0
\end{array}\right)\right| \\
& =\left|\begin{array}{cc}
0 & n(x) S R_{x} L_{y \theta}-n(g) n(y) S R_{y x} \\
n(g) n(y) S L_{y x}-n(x) S L_{x} R_{g y} & 0
\end{array}\right| \\
& =\left|R_{x} L_{x}\right| \cdot\left|n(x) L_{y o}-n(g) n(y) R_{y}\right| \cdot\left|n(g) n(y) L_{y}-n(x) R_{o y}\right|
\end{aligned}
$$

[2, Lemma 1].

- The Cayley-Dickson process (the case $g=\gamma$, a scalar) may be eliminated by this argument too. If $\gamma \geqq 0$, let $y=\beta$ in $\Re, n(x)=\gamma \beta^{2}$; if $\gamma<0$, let $y=i, f=j, n(x)=-\gamma$ in what follows. 
since $x, y, g$ are quaternions. That is, $\left|n(x) L_{y 0}-n(g) n(y) R_{y}\right|=0$ would imply that transformation (2) is singular.

Choose $f=\zeta y+y g$. Then $f\left\{n(x) L_{y g}-n(g) n(y) R_{y}\right\}=n(x) \zeta y g y$ $+n(x) y g y g-n(g) n(y) \zeta y^{2}-n(g) n(y) y g y=n(x) y^{2} g S \cdot g-n(g) n(x) y^{2}=0$. Hence (2) is singular and $A$ is not a division algebra over $\Re$.

The easy generalization that there is no choice of $g$ to make $A$ a division algebra of order 16 over any field $F$ should not be made. For the singularity of transformation (2) implies that there exists an element $h \neq 0$ in $C$ such that $n(x)\{(h x) g\} y=n(g) n(y) h(x y)$. Since the norm of a product is the product of the norms in an alternative division algebra, ${ }^{6}$

$$
\overline{n(x)^{3}} n(h) n(g) n(y)=\overline{n(g)^{2}} \overline{n(y)^{3}} n(h) n(x) \text { or } \overline{n(x)^{2}}=n(g) \overline{n(y)^{2}}
$$

in case $g \neq 0$. That is, the transformation (2) cannot be singular (and $A$ is therefore a division algebra) for any choice of $g$ in $C$ such that $n(g)$ is not the square of an element in $F$.

For example, let $F$ be in particular the field $R$ of rational numbers, and $g=1+i$ so that $n(g)=2$. Then the algebra $A$ with multiplication defined by (1) is a division algebra of order 16 over $R$.

\section{REFERENCES}

1. A. A. Albert, Non-associative algebras. I. Fundamental concepts and isotopy, Ann. of Math. (2) vol. 43 (1942) pp. 685-708.

2. R. D. Schafer, Alternative algebras over an arbitrary field, Bull. Amer. Math. Soc. vol. 49 (1943) pp. 549-555.

San Francisco, Calif.

- [2, Lemma 2]. 